\title{
A FAST RADIO BURST IN THE DIRECTION OF THE CARINA DWARF SPHEROIDAL GALAXY
}

\author{
V. RAVI ${ }^{1,2,3}$, R. M. SHANNON ${ }^{2}$, AND A. JAMESON ${ }^{3}$ \\ ${ }^{1}$ School of Physics, University of Melbourne, Parkville, VIC 3010, Australia; v.vikram.ravi@ gmail.com \\ ${ }^{2}$ CSIRO Astronomy and Space Science, Australia Telescope National Facility, P.O. Box 76, Epping, NSW 1710, Australia \\ ${ }^{3}$ Swinburne University of Technology, Centre for Astrophysics and Supercomputing, Mail H39, P.O. Box 218, VIC 3122, Australia \\ Received 2014 October 28; accepted 2014 December 4; published 2015 January 14
}

\begin{abstract}
We report the real-time discovery of a fast radio burst (FRB 131104) with the Parkes radio telescope in a targeted observation of the Carina dwarf spheroidal galaxy. The dispersion measure of the burst is $779 \mathrm{~cm}^{-3} \mathrm{pc}$, exceeding predictions for the maximum line-of-sight Galactic contribution by a factor of 11 . The temporal structure of the burst is characterized by an exponential scattering tail with a timescale of $2.0_{-0.5}^{+0.8} \mathrm{~ms}$ at $1582 \mathrm{MHz}$ that scales as frequency to the power $-4.4_{-1.8}^{+1.6}$ (all uncertainties represent $95 \%$ confidence intervals). We bound the intrinsic pulse width to be $<0.64 \mathrm{~ms}$ due to dispersion smearing across a single spectrometer channel. Searches in $78 \mathrm{hr}$ of follow-up observations with the Parkes telescope reveal no additional sporadic emission and no evidence for associated periodic radio emission. We hypothesize that the burst is associated with the Carina dwarf galaxy. Follow-up observations at other wavelengths are necessary to test this hypothesis.
\end{abstract}

Key words: galaxies: individual (Car dSph) - intergalactic medium - pulsars: general radio continuum: general - scattering

\section{INTRODUCTION}

The discovery of fast radio bursts (FRBs) presents a potentially transformational challenge to the understanding of the ephemeral Universe. FRBs are characterized by bright ( $\sim \mathrm{Jy}$ peak flux densities) millisecond-duration pulses with inferred dispersion measures (DMs) that significantly exceed (by factors of 3-20) those expected from the Galaxy. Seven FRBs have recently been found in surveys at $\sim 1.4 \mathrm{GHz}$ with both the Parkes (Lorimer et al. 2007; Thornton et al. 2013; Burke-Spolaor \& Bannister 2014) and Arecibo (Spitler et al. 2014) telescopes.

The intrinsic pulse durations, with some upper limits of $\lesssim 1 \mathrm{~ms}$, imply coherent emission originating from compact regions (Katz 2014a; Luan \& Goldreich 2014). No additional bursts were detected in follow-up observations of one FRB (Lorimer et al. 2007), although these observations were conducted six years after the event. While FRBs have similar dispersion characteristics to the apparently terrestrial perytons (BurkeSpolaor et al. 2011; Kocz et al. 2012; Saint-Hilaire et al. 2014; Kulkarni et al. 2014), they can be distinguished through their isolation on the sky, preferred locations away from the bulk of the Galactic disk (Burke-Spolaor \& Bannister 2014), and broadband nature.

Any explanation for FRBs must account for the DM excesses, $\Delta \mathrm{DM}$, over the maximum line-of-sight DMs predicted by models for the Galactic electron density (Cordes \& Lazio 2002). Within the Milky Way, the large values of $\Delta D M$ have been explained by invoking photo- or shock-ionized nebulae (Kulkarni et al. 2014), or emission from deep within stellar coronae (Loeb et al. 2014). However, these explanations are inconsistent, respectively, with constraints from $\mathrm{H} \alpha$ observations on the presence of dense ionized nebulae (Kulkarni et al. 2014), and with the lack of observed deviations from the cold plasma dispersion law (Tuntsov 2014; Katz 2014b; Dennison 2014).

If the Galactic electron density models are credible, and the frequency-dependent delays of FRBs are not intrinsic to their sources, it is possible that the DM excesses are caused by FRBs being extragalactic. In this case, the distances inferred from the values of $\Delta \mathrm{DM}$ are $\gtrsim 1 \mathrm{Gpc}$ in the absence of significant host contributions (Ioka 2003). A menagerie of exotic extragalactic sources of FRBs have been theorized, including the collapse of gravitationally unstable magnetars to black holes (Falcke \& Rezzolla 2014), giant magnetar flares (Lyubarsky 2014), and emission from superconducting cosmic strings (Vachaspati 2008). Despite some predictions (e.g., Ravi \& Lasky 2014), no astrophysical transient events at other wavelengths, such as supernovae or gamma-ray bursts, have yet been associated with FRBs.

The large positional uncertainties of $>3.5$ at Arecibo and $>14^{\prime}$ at Parkes have made it impossible to uniquely associate FRBs with any objects. Also, all FRBs have hitherto been detected in post-processing of data from blind radio surveys of large areas of the sky, and have therefore not been rapidly re-observed.

Here, we report the real-time discovery of a new FRB (131104) with the Parkes telescope. The observations leading to the discovery of FRB 131104, which we detail in Section 2, were targeted at the Carina dwarf spheroidal (Car dSph) satellite of the Milky Way. An analysis of the temporal and spectral structure of the burst is presented in Section 3. We describe our immediate follow-up observations in Section 4, discuss the implications of our results in Section 5, and summarize in Section 6.

\section{DISCOVERY OBSERVATION}

We conducted our observations on UT 2013 November 4 (MJD 56600) with the 13 beam $21 \mathrm{~cm}$ multibeam (MB) receiver (Staveley-Smith et al. 1996) at the prime focus of the $64 \mathrm{~m}$ Parkes antenna. The FWHM of all beam responses on the sky are approximately $14^{\prime}$, although we note that the response patterns of the outer beams are mildly elliptical. Data were recorded with the Berkeley-Parkes-Swinburne Recorder (BPSR) digital spectrometer using the same configuration used for the High Time Resolution Universe survey (Keith et al. 2010). For each of the $13 \mathrm{MB}$ beams, 8 bit 1024 channel spectra were integrated for $64 \mu$ s intervals within a $400 \mathrm{MHz}$ band centered on $1382 \mathrm{MHz}$. Data from each of the linearly polarized feeds were summed to form total-intensity time series. 


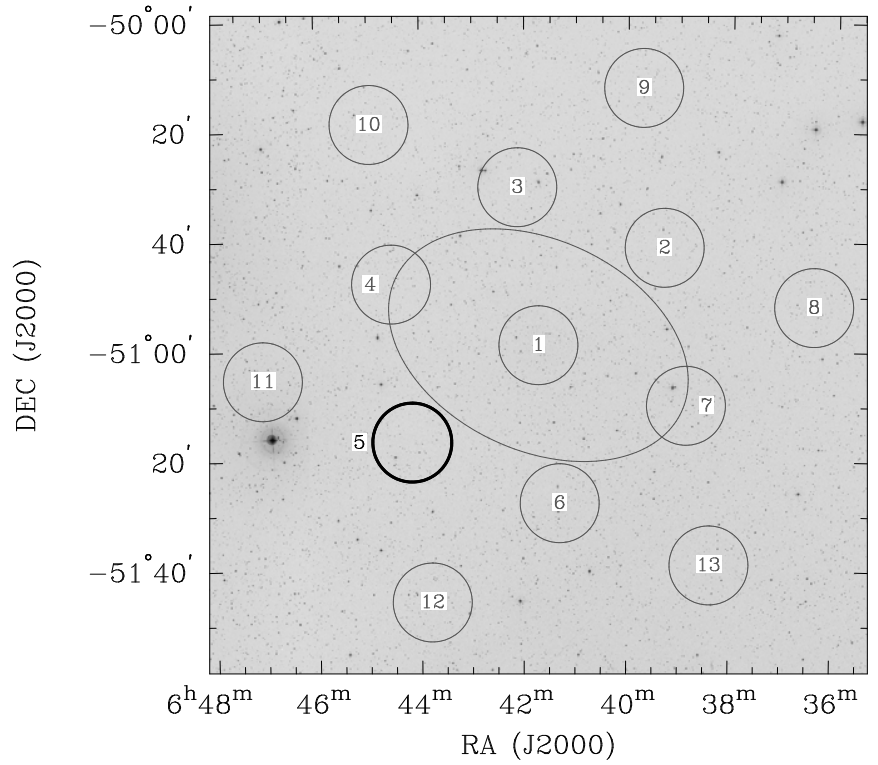

Figure 1. Positions of the Parkes MB beams, labeled 1-13 and displayed as $14^{\prime}$ diameter circles, when FRB 131104 was detected. The FRB was only detected in beam 5 (thick black circle). The tidal ellipse of Car dSph is also shown (Irwin \& Hatzidimitriou 1995), and the background inverted grayscale image is taken from the Second Digitized Sky Survey red images.

A real-time transient search pipeline, HEIMDALL ${ }^{4}$ (Barsdell et al. 2012), was in operation on the BPSR computing cluster during the observations. This pipeline was used to search for isolated pulses at DMs between $1.5-2000 \mathrm{~cm}^{-3} \mathrm{pc}$ with widths up to $0.262 \mathrm{~s}$. Events within our search ranges that were likely caused by radio-frequency interference (RFI) were excised in real time (see, e.g., Burke-Spolaor \& Bailes 2010). Specifically, events that were coincident in time with non-dispersed events or with ones in three or more other beams were flagged as RFI. We tested the entire observing setup by pointing each beam in turn at the bright millisecond pulsar PSR J0437-4715 to ensure that single pulses were being detected with the expected signalto-noise ratios (S/Ns). We also carried out a $1 \mathrm{hr}$ observation of PSR B0540-69 in the Large Magellanic Cloud, which is the most distant known emitter of giant pulses (Johnston \& Romani 2003). We detected one such pulse with a S/N of 7 at the known $\mathrm{DM}$ of 146.5. This detection is consistent with the measured rate of approximately two per hour.

We then conducted a series of $1 \mathrm{hr}$ observations with the central MB beam positioned on Car $\mathrm{dSph}$. We disabled parallactic angle tracking for the MB feed system; consequently the outer MB beams rotated slowly on the sky. At 18:04:01.2 UT, approximately 21 min following the start of the second observation of Car dSph, the HEIMDALL pipeline reported the detection of a transient event at a DM of $779 \mathrm{~cm}^{-3} \mathrm{pc}$, with a $\mathrm{S} / \mathrm{N}$ of 30.6 when the time series was smoothed using a boxcar of width $2.08 \mathrm{~ms}$. The event was detected in beam 5 of the MB receiver, which at the time was pointed at the celestial coordinates (J2000) $06^{\mathrm{h}} 44^{\mathrm{m}} 10^{\mathrm{s}} .4,-51^{\mathrm{d}} 16^{\mathrm{m}} 40^{\mathrm{s}}$. The positions of all the beams on the sky when the event was detected are shown in Figure 1. Data recorded from beam 5 corresponding to the event are shown as a dynamic spectrum in Figure 2.

We did not identify any significant events (with $\mathrm{S} / \mathrm{N}>6$ ) in the other beams that were coincident in time with the event

\footnotetext{
4 The source code for this pipeline is publicly available at
} http://sourceforge.net/projects/heimdall-astro.

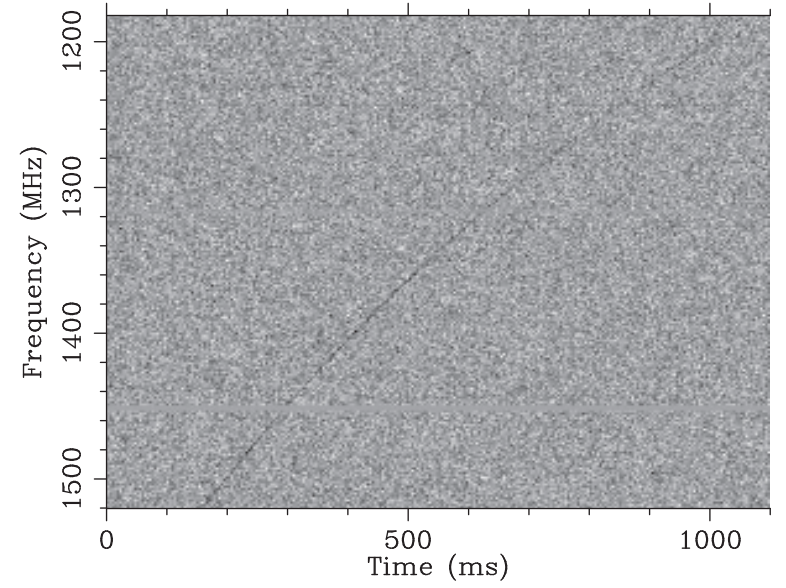

Figure 2. Dynamic spectrum of FRB 131104, collapsed to a frequency resolution of $1.5625 \mathrm{MHz}$ and a time resolution of $4.1 \mathrm{~ms}$. The inverted grayscale intensity map represents the detected radio power in arbitrary units. Some persistent narrow-band RFI has been blanked.

in beam 5. We also inspected the summed time series from all other beams dedispersed using the DM estimate from HEIMDALL of $779 \mathrm{~cm}^{-3} \mathrm{pc}$, and again found no significant coincident occurrences. No coincident events were present when summed time series were formed using pairs of beams immediately surrounding beam 5 .

The background of significant candidate events with DMs greater than our threshold of $1.5 \mathrm{~cm}^{-3} \mathrm{pc}$ identified with the HEIMDALL pipeline during the discovery observation was characteristic of times not especially affected by RFI. These candidates typically had widths greater than $100 \mathrm{~ms}$, and an approximately log-uniform distribution of DMs throughout the searched range. The event of interest was the highest $\mathrm{S} / \mathrm{N}$ occurrence during the discovery observation.

The detected event is similar to the FRBs of Thornton et al. (2013), Spitler et al. (2014), and Burke-Spolaor \& Bannister (2014), and is markedly different from peryton emission (BurkeSpolaor et al. 2011). Unlike perytons, and most forms of RFI, the event was isolated to a single beam of the MB receiver. The DM estimate from HEIMDALL is much larger than the apparent DM of any published peryton, and exceeds the expected Galactic contribution along this line of sight by $\Delta \mathrm{DM}=710 \mathrm{~cm}^{-3} \mathrm{pc}$ (Cordes \& Lazio 2002). An inspection of Figure 2 shows evidence for the presence of emission across the entire observed frequency band, in contrast to perytons, and the small derived width from HEIMDALL of $\sim 2 \mathrm{~ms}$ is also significantly lower than that of perytons. Further, while perytons predominantly manifest during the day (Burke-Spolaor et al. 2011; Kocz et al. 2012; Saint-Hilaire et al. 2014), our detection occurred at 05:04 local time, prior to sunrise. We hence conclude that the event is a FRB, designated FRB 131104.

\section{ANALYSIS OF THE TEMPORAL AND SPECTRAL STRUCTURE}

The temporal profile of FRB 131104, displayed in Figure 3, shows evidence for changes in both amplitude and width as functions of the radio frequency. In contrast to the modeling of the frequency-dependent delays and shapes of previous FRBs (Lorimer et al. 2007; Thornton et al. 2013; Spitler et al. 2014; Burke-Spolaor \& Bannister 2014), we conducted a Bayesian analysis of the total intensity time series to measure the burst physical parameters. This approach allowed us to 
Table 1

Details of Time-frequency Fits (All Uncertainties are the 95\% Confidence Intervals)

\begin{tabular}{|c|c|c|c|c|c|c|c|c|c|}
\hline Model & $\begin{array}{c}c_{1232} \\
(\mathrm{Jy} \mathrm{ms})\end{array}$ & $\begin{array}{c}c_{1332} \\
\text { (Jy ms) }\end{array}$ & $\begin{array}{c}c_{1432} \\
(\mathrm{Jy} \mathrm{ms})\end{array}$ & $\begin{array}{c}c_{1532} \\
(\mathrm{Jy} \mathrm{ms})\end{array}$ & $\begin{array}{c}\mathrm{DM} \\
\left(\mathrm{cm}^{-3} \mathrm{pc}\right)\end{array}$ & $\begin{array}{c}\tau_{s} \\
(\mathrm{~ms})\end{array}$ & $\alpha$ & $\begin{array}{l}t_{0} \\
(\mathrm{~s})\end{array}$ & $\Delta \mathrm{BIC}$ \\
\hline 0 & $1.9 \pm 0.2$ & $2.0_{-0.2}^{+0.1}$ & $2.7 \pm 0.2$ & $3.7_{-0.3}^{+0.4}$ & $779.1_{-0.2}^{+0.1}$ & $\ldots$ & $\cdots$ & $1.03435_{-8 \times 10^{-5}}^{+7 \times 10^{-5}}$ & 0 \\
\hline 1 & $2.3_{-0.3}^{+0.2}$ & $2.0_{-0.1}^{+0.2}$ & $2.3_{-0.2}^{+0.1}$ & $3.0_{-0.4}^{+0.3}$ & $779.0_{-0.2}^{+0.2}$ & $1.1_{-0.1}^{+0.1}$ & $\ldots$ & $1.0338_{-1 \times 10^{-4}}^{+1 \times 10^{-4}}$ & -299 \\
\hline 2 & $2.5_{-0.3}^{+0.4}$ & $2.0 \pm 0.2$ & $2.2_{-0.2}^{+0.1}$ & $2.6 \pm 0.4$ & $778.5_{-0.3}^{+0.2}$ & $2.0_{-0.5}^{+0.8}$ & $4.4_{-1.8}^{+1.6}$ & $1.0340_{-1 \times 10^{-4}}^{+1 \times 10^{-4}}$ & -306 \\
\hline
\end{tabular}

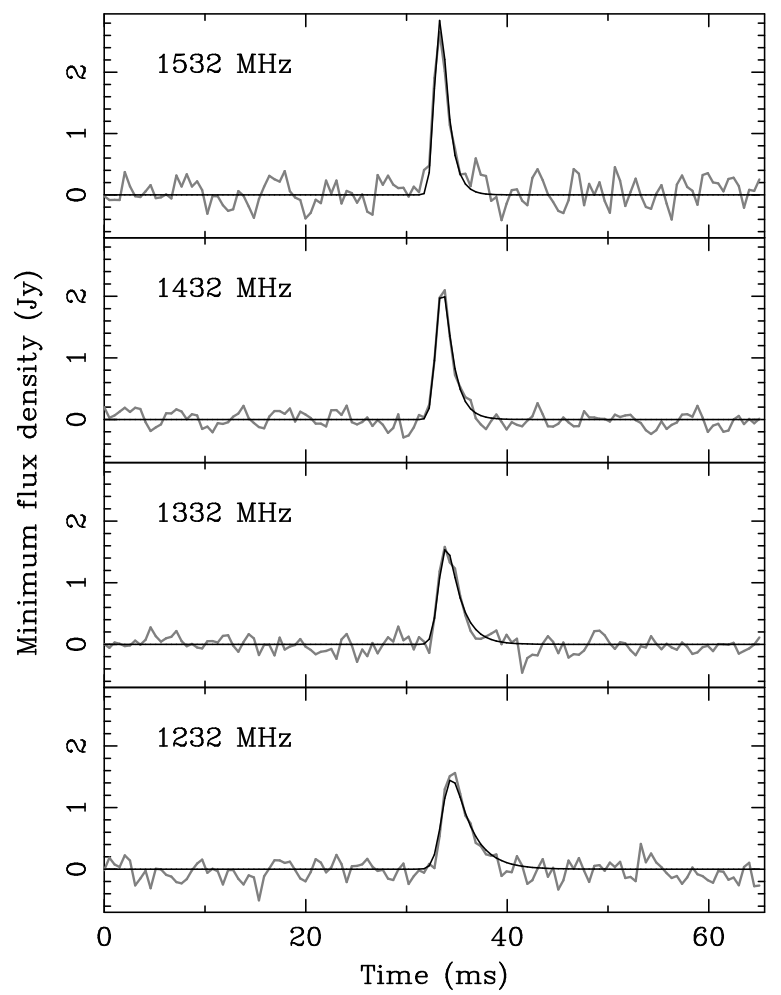

Figure 3. Comparison between the best-fitting model (black) and the frequencydependent profile (gray) of FRB 131104. The data were dedispersed at the bestfit DM listed in Table 1, split into $100 \mathrm{MHz}$ bands centered on the displayed frequencies, and summed to a time-resolution of $0.512 \mathrm{~ms}$. The flux density scale is based on our on-sky noise temperature measurements for beam 5 of the Parkes MB receiver.

fully account for covariances between model parameters, and to obtain accurate confidence intervals for our parameter estimates. We used the Bayes Information Criterion (BIC; Schwarz 1978) to perform model selection and justify the successive inclusion of free parameters. The BIC for a given model is $-2 \ln \hat{L}+k[\ln (n)-\ln (2 \pi)]$, where $\hat{L}$ is the likelihood estimate, $k$ is the number of model parameters, and $n$ is the number of measurements. If adding a new free parameter to a model reduces the BIC, the new model is accepted because the likelihood increase is not simply due to the addition of the new parameter.

In order to accelerate the analysis, the data were divided into 16 sub-bands across the full $400 \mathrm{MHz}$ band. The two uppermost in frequency were discarded because instrumental filtering of RFI from the Thuraya 3 satellite (Keith et al. 2010) rendered them unusable. The FRB was detectable by eye in all other bands. We then dedispersed the data in each of these remaining bands using the initial DM estimate of $779 \mathrm{~cm}^{-3} \mathrm{pc}$ to form 14 time series, while retaining the dispersion delays between each band. Adjacent samples in each time series were summed to a time-resolution of $0.512 \mathrm{~ms}$. Finally, through visual inspection of these time series, we selected windows of width $15.36 \mathrm{~ms}$ containing all the evident signal, and used these measurements as the basis for our analysis.

We assessed the noise properties of the time-series by examining $2 \mathrm{~s}$ of data immediately adjacent to these windows in each frequency sub-band. We found that the values were consistent with normal distributions. We therefore measured the variances of these time series and assumed that the noise during the $15.36 \mathrm{~ms}$ windows was statistically identical.

We considered a hierarchical series of models for the pulse morphology. The simplest model consisted of a Dirac delta function impulse, dispersion-smeared at each frequency over a bandwidth corresponding to the BPSR frequency resolution of $0.391 \mathrm{MHz}$. We modeled the smeared pulse profile as a Gaussian function, which approximately corresponds to the frequency response of the two-tap digital filterbank, and made the conventional assumption of cold plasma dispersion. In this case, the signal intensity $S$ at a given time $t$ and frequency $v$ was therefore:

$$
S(t, v)=\frac{A(v)}{\sqrt{2 \pi \sigma_{\mathrm{DM}}^{2}(v)}} \exp \left[\frac{-\left(t-t_{0}-\tau_{\mathrm{DM}}(v)\right)^{2}}{\sigma_{\mathrm{DM}}^{2}(v)}\right],
$$

where $t_{0}$ is a reference time at a reference frequency of $v_{0}=1582 \mathrm{MHz}$,

$$
\tau_{\mathrm{DM}}(v)=(4.15 \mathrm{~ms}) \mathrm{DM}\left[(v / \mathrm{GHz})^{-\beta}-\left(v_{0} / \mathrm{GHz}\right)^{-\beta}\right]
$$

with $\beta=2$, and

$$
\sigma_{\mathrm{DM}}(v)=\left(1.622 \times 10^{-3} \mathrm{~ms}\right) \mathrm{DM}(v / \mathrm{GHz})^{-\beta-1} .
$$

Finally, we modeled the fluence, $A(v)$, in four separate $100 \mathrm{MHz}$ bands within the full observing bandwidth; in order of increasing frequency, we denote these fluences by $c_{1232}, c_{1332}, c_{1432}$, and $c_{1532}$. We evaluated the six free parameters of this model by exploring the likelihood space using the emcee Markov Chain Monte Carlo software package (Foreman-Mackey et al. 2013). In the first row of Table 1 we present the maximum-likelihood parameter values and their $95 \%$ confidence intervals for this model, which we term Model 0.

While in our fitting procedure we expressed the fluences as $\mathrm{S} / \mathrm{Ns}$ in each sub-band, we present them in physical units of Jy ms in Table 1. The conversions are based on our on-sky noise temperature measurements for beam 5 of the Parkes MB receiver using the unresolved radio source Hydra $A$, and under the assumption that the FRB originated at the boresight of the beam. Our fluence measurements are therefore lower limits.

We extended this model by convolving the Gaussian function in Equation (1) with a one-sided exponential with timescale

$$
\tau(\nu)=\tau_{s}\left(\nu / \nu_{0}\right)^{-\alpha},
$$


where we initially assumed $\alpha=0$, and where $\tau_{s}$ is a free parameter. This allows for the pulse to be modeled with an exponential tail. This model, denoted by Model 1 in Table 1, significantly reduced the BIC with respect to Model 0 . A model where we instead replaced $\sigma_{\mathrm{DM}}(v)$ in Equation (1) with $\left[\sigma^{2}+\left(\sigma_{\mathrm{DM}}(v)-\sigma_{\mathrm{DM}}\left(v_{0}\right)\right)^{2}\right]^{1 / 2}$, where $\sigma$ is a free parameter, did not produce as great a reduction in the BIC. A model which instead included $\beta$ as a free parameter also did not produce as great a reduction in the BIC.

We then allowed $\alpha$ to be a free parameter in addition to $\tau_{s}$, enabling a search for scattering as an origin for the exponential tail. This caused a further reduction in the BIC. The resulting parameters are also listed in Table 1, and denoted by Model 2. Neither the addition of $\sigma$ nor the addition of $\beta$ as a free parameter further reduced the BIC. We hence have no evidence for a pulse half-width, $\sigma$, at $v=v_{0}$ that is different to the dispersion smearing timescale $\sigma_{\mathrm{DM}}\left(v_{0}\right)=0.32 \mathrm{~ms}$. We also have no evidence for $\beta \neq 2$.

In contrast, we find strong evidence for frequency-broadening of the pulse profile. The broadening timescale is $\tau_{s}=2.0_{-0.5}^{+0.8} \mathrm{~ms}$ at $1582 \mathrm{MHz}$, and the index of the frequency-dependence is $\alpha=4.4_{-1.8}^{+1.6}$ (all uncertainties are the $95 \%$ confidence intervals). This is the second time that a FRB has been shown to exhibit such an exponential tail (after FRB 110220; Thornton et al. 2013), although other studies have found that FRB effective widths have similar frequency dependencies (Lorimer et al. 2007; Thornton et al. 2013; Burke-Spolaor \& Bannister 2014). The value of $\alpha$ corresponds to scattering by a Kolmogorovturbulent plasma (in which case $\alpha=4.4$ is expected; e.g., Rickett 1977), and is consistent with both scattering in a diffuse, extended medium, or in a geometrically thin region along the line of sight, or both.

Based on the measurements of the fluences, we find $A(v) \propto$ $v^{0.3 \pm 0.9}$. This marginally inverted spectrum is broadly consistent with the analysis of FRB 121102 detected with the Arecibo telescope by Spitler et al. (2014). We note that, depending on the location of the FRB within the primary beam, both hardening and softening of the spectrum can be induced.

\section{FOLLOW-UP OBSERVATIONS}

We re-observed the field of FRB 131104 for three hours immediately following its occurrence, continuously rotating the receiver to keep beam 5 directed toward the FRB position. We also observed the field for $6 \mathrm{hr}$ lengths during the subsequent three days, and for an additional $57 \mathrm{hr}$ at arbitrary times during the following year. With the exception of RFI, we did not detect any other event with $\mathrm{DM}>1.5 \mathrm{~cm}^{-3} \mathrm{pc}$, a width of $\leqslant 65 \mathrm{~ms}$, and $\mathrm{S} / \mathrm{N}>8$ in any of the MB beams. The upper width threshold aided in rejecting the majority of spurious candidates.

Given the all-sky FRB rate estimated by Thornton et al. (2013), we would have expected to see 0.5 FRBs in the totality of our observations. Assuming that the occurrence of FRBs can be modeled as an isotropic Poisson process, the probability of finding one or more FRBs in all our observations is 0.4 .

We also searched for periodic radio emission in the FRB discovery observation, and in four $0.5 \mathrm{hr}$ follow-up observations of the apparent FRB position spaced evenly over six months. We used standard pulsar search software to search both at the $\mathrm{DM}$ of the FRB and at all DMs $>1.5$. No periodic signal was found with $\mathrm{S} / \mathrm{N}>8$ and a period between $1 \mathrm{~ms}$ and $10 \mathrm{~s}$ that was not attributable to RFI. For a long-period pulsar with a duty cycle of $10 \%$, the limiting sensitivity of our search at each epoch was $0.1 \mathrm{mJy}$.

\section{DISCUSSION}

FRB 131104 is the first FRB to be found in a targeted search for such events. Car dSph, with a moderate Galactic latitude ( $b=22.2$ ), is unique among dwarf Milky Way companions in having undergone three widely spaced episodes of star formation (Hurley-Keller et al. 1998), and the oldest stellar population shows some evidence for tidal disruption (Battaglia et al. 2012; McMonigal et al. 2014). We chose to observe Car dSph because of the low expected Galactic contribution to the DM in its direction, the presence of multiple stellar populations and possible tidal debris, the fact that the Lorimer et al. (2007) FRB was detected near the Small Magellanic Cloud, and the possibility of discovering pulsars in Car dSph in order to identify the Local Group contribution to DM along this sightline. Despite the inferred FRB rate of $10^{4} \mathrm{sky}^{-1} \mathrm{day}^{-1}$ (Thornton et al. 2013) and the total effective Parkes field-of-view of $\sim 2 \times 10^{-5} \times 4 \pi$ sr, this event remarkably occurred within $1.5 \mathrm{hr}$ of the beginning of our observations of Car $\mathrm{dSph}$.

The location of beam 5 of the MB receiver when FRB 131104 was detected (Figure 1) is also coincident with stellar debris associated with the Large Magellanic Cloud (McMonigal et al. 2014), which is a factor of $\sim 2$ closer to the Earth than Car dSph. However, the proximity of the half-power beam point of beam 5 to Car dSph tidal ellipse means that FRB 131104 may indeed be coincident in sky location with that galaxy.

However, for FRB 131104 to have originated within Car $\mathrm{dSph}$, which is $101 \pm 5 \mathrm{kpc}$ distant from the solar system (Mateo 1998), its source would have to be located behind, or embedded in, an overdensity of ionized interstellar gas relative to the Milky Way ionized halo. Recent estimates for the electron density of the Milky Way ionized halo suggest $n_{e}=(2 \pm 0.6) \times 10^{-4} \mathrm{~cm}^{-3}$ (Gupta et al. 2012). Even if the DM contribution from Car dSph is $100 \mathrm{~cm}^{-3} \mathrm{pc}$, the intervening medium would need to have a mean electron density of $n_{e} \sim 6 \times 10^{-3} \mathrm{~cm}^{-3}$.

Such large densities have, however, been measured in the Magellanic stream (e.g., Fox et al. 2014), a part of which lies along the Car dSph sightline. FRB 010724 Lorimer et al. (2007), observed just a few degrees South of the Small Magellanic Cloud, had a sightline that was clearly associated with the Magellanic stream. Further detailed observational analyses of the ionized gas content surrounding the Milky Way along the line of sight to FRB 131104 would reveal whether there is sufficient material to associate this FRB with Car dSph.

Pending such investigations, we cannot constrain the distance to FRB 131104. Hence, various possibilities for the origin of FRB 131104 remain open. If FRB 131104 originated from the close surrounds of the Milky Way, it could conceivably represent a form of giant pulse emission. The brightest such event detected from the Crab pulsar with the Arecibo telescope at $0.43 \mathrm{GHz}$ would have had a $\mathrm{S} / \mathrm{N}$ of $10^{6.5}$ in the absence of radio emission from the Crab nebula (Cordes et al. 2004). Assuming a flux density spectrum $\propto v^{-3}$ for giant pulses, such an event would have been detectable with Parkes at the distance to Car dSph with a $\mathrm{S} / \mathrm{N}$ of $\sim 26$. Furthermore, the energy distribution of giant pulses from the Crab pulsar appears to flatten at the high fluence end (Cordes et al. 2004; Mickaliger et al. 2012), suggesting that even brighter pulses may yet be found than the Cordes et al. (2004) event. Such a scenario for FRB 131104 will be best confirmed through an independent estimate of the line-of-sight DM to Car dSph, and through the observation of a repeat event. 


\section{SUMMARY}

FRB 131104 was detected in a targeted observation of the Milky Way satellite Car dSph with the Parkes radio telescope. It is possible that the large dispersion of the burst may be partly associated with circum-Galactic ionized gas along the line of sight. The pulse was found to have both an exponential tail and an inverted spectrum; the frequency dependence of the tail is consistent with scattering in a turbulent medium. The realtime detection of FRB 131104 enabled rapid follow-up with the Parkes telescope, which revealed no sporadic or periodic radio emission. Further analyses of circum-Galactic ionized gas along the sightline to FRB 131104 are required to constrain the distance and origin of this FRB.

We thank M. Bailes and E. Petroff for useful discussions, and the Swinburne pulsar group for making their real-time single pulse detector available for this experiment. We also thank the Parkes Observatory staff for hospitality during our visits. The Parkes Radio Telescope is part of the Australia Telescope, which is funded by the Commonwealth of Australia for operation as a National Facility by CSIRO. We acknowledge the use of NASA's SkyView facility (http://skyview.gsfc.nasa.gov) located at NASA Goddard Space Flight Center. This work was performed on the $g$ STAR national facility at Swinburne University of Technology. $g$ Star is funded by Swinburne and the Australian Government's Education Investment Fund.

\section{REFERENCES}

Barsdell, B. R., Bailes, M., Barnes, D. G., \& Fluke, C. J. 2012, in ASP Conf. Ser. 461, Astronomical Data Analysis Software and Systems XXI, ed. P. Ballester, D. Egret, \& N. P. F. Lorente (San Francisco, CA: ASP), 37

Battaglia, G., Irwin, M., Tolstoy, E., de Boer, T., \& Mateo, M. 2012, ApJL, 761, L31

Burke-Spolaor, S., \& Bailes, M. 2010, MNRAS, 402, 855
Burke-Spolaor, S., Bailes, M., Ekers, R., Macquart, J.-P., \& Crawford, F., III. 2011, ApJ, 727, 18

Burke-Spolaor, S., \& Bannister, K. W. 2014, ApJ, 792, 19

Cordes, J. M., Bhat, N. D. R., Hankins, T. H., McLaughlin, M. A., \& Kern, J. 2004, ApJ, 612, 375

Cordes, J. M., \& Lazio, T. J. W. 2002, arXiv:astro-ph/0207156

Dennison, B. 2014, MNRAS, 443, L11

Falcke, H., \& Rezzolla, L. 2014, A\&A, 562, A137

Foreman-Mackey, D., Hogg, D. W., Lang, D., \& Goodman, J. 2013, PASP, 125,306

Fox, A. J., Wakker, B. P., Barger, K. A., et al. 2014, ApJ, 787, 147

Gupta, A., Mathur, S., Krongold, Y., Nicastro, F., \& Galeazzi, M. 2012, ApJL, 756, L8

Hurley-Keller, D., Mateo, M., \& Nemec, J. 1998, AJ, 115, 1840

Ioka, K. 2003, ApJL, 598, L79

Irwin, M., \& Hatzidimitriou, D. 1995, MNRAS, 277, 1354

Johnston, S., \& Romani, R. W. 2003, ApJL, 590, L95

Katz, J. I. 2014a, PhRvD, 89, 103009

Katz, J. I. 2014b, ApJ, 788, 34

Keith, M. J., Jameson, A., van Straten, W., et al. 2010, MNRAS, 409, 619

Kocz, J., Bailes, M., Barnes, D., Burke-Spolaor, S., \& Levin, L. 2012, MNRAS, 420, 271

Kulkarni, S. R., Ofek, E. O., Neill, J. D., Zheng, Z., \& Juric, M. 2014, ApJ, 797, 70

Loeb, A., Shvartzvald, Y., \& Maoz, D. 2014, MNRAS, 439, L46

Lorimer, D. R., Bailes, M., McLaughlin, M. A., Narkevic, D. J., \& Crawford, F. 2007, Sci, 318, 777

Luan, J., \& Goldreich, P. 2014, ApJL, 785, L26

Lyubarsky, Y. 2014, MNRAS, 442, L9

Mateo, M. L. 1998, ARA\&A, 36, 435

McMonigal, B., Bate, N. F., Lewis, G. F., et al. 2014, MNRAS, 444, 3139

Mickaliger, M. B., McLaughlin, M. A., Lorimer, D. R., et al. 2012, ApJ, 760,64

Ravi, V., \& Lasky, P. D. 2014, MNRAS, 441, 2433

Rickett, B. J. 1977, ARA\&A, 15, 479

Saint-Hilaire, P., Benz, A. O., \& Monstein, C. 2014, ApJ, 795, 19

Schwarz, G. E. 1978, AnSta, 6, 461

Spitler, L. G., Cordes, J. M., Hessels, J. W. T., et al. 2014, ApJ, 790, 101

Staveley-Smith, L., Wilson, W. E., Bird, T. S., et al. 1996, PASA, 13, 243

Thornton, D., Stappers, B., Bailes, M., et al. 2013, Sci, 341, 53

Tuntsov, A. V. 2014, MNRAS, 441, L26

Vachaspati, T. 2008, PhRvL, 101, 141301 